\title{
Perfil psicosocial de adolescentes españoles agresores y víctimas de violencia de pareja ${ }^{*}$
}

\section{Psychosocial Profile of Spanish Adolescent Aggressors and Victims of} Dating Violence

Recepción: 15 Septiembre 2016 | Aceptación: 21 Febrero 2018

\author{
Laura Carrascosa ${ }^{\mathrm{a}}$ \\ Universidad de Valencia, España \\ ORCID: http://orcid.org/0000-0002-3392-4678 \\ María-Jesús CaVA \\ Universidad de Valencia, España \\ Sofia Buelga \\ Universidad de Valencia, España
}

a Autor de correspondencia. Correo electrónico:

Laura.Carrascosa@uv.es

Para citar este artículo: Carrascosa, L., Cava, M. J., \& Buelga, S. (2018). Perfil psicosocial de adolescentes españoles agresores y víctimas de violencia de pareja. Universitas Psychologica, 17(3), 1-10. https://doi.org/1 0.11144/Javeriana.upsy17-3.ppae

\section{RESUMEN}

El objetivo de este estudio fue analizar posibles diferencias en satisfacción con la vida, ánimo depresivo, autoconcepto y comunicación familiar entre adolescentes no implicados en violencia de pareja, agresores, víctimas, implicados en el rol de agresor-víctima de forma ocasional e implicados en este rol frecuentemente. Participaron 672 adolescentes españoles (325 chicas, 347 chicos), entre 12 y 19 años $(\mathrm{M}=14.45$; DT $=1.62)$. Los resultados muestran un perfil psicosocial más negativo en las chicas víctimas de violencia de pareja y en los chicos implicados frecuentemente en el rol de agresor-víctima. En las chicas con mayor implicación en violencia de pareja se observan también mayores dificultades en la comunicación familiar. Estos resultados muestran la existencia de perfiles psicosociales diferentes en chicos y chicas, que desempeñan diferentes roles en violencia de pareja, y la utilidad de considerar estos perfiles en el desarrollo de programas de intervención.

Palabras clave

violencia de pareja; victimización; adolescencia; perfil psicosocial.

\section{ABSTRACT}

The objective of this study was to analyze possible differences in life satisfaction, depressive mood, self-concept and family communication among adolescents not involved in dating violence, bullies, victims, occasionally involved in a role of aggressor-victim and frequently involved in a role of aggressor-victim were analyzed. 672 Spanish adolescents (325 girls, 347 boys) between 12 and 19 years $(\mathrm{M}=14.45, \mathrm{SD}=$ 1.62) participated in this study. The results show a more negative psychosocial profile in girls victims of dating violence and boys frequently involved in the role of aggressor-victim. More difficulties in family communication were also observed in girls with high involvement in dating violence. These results show the existence of different psychosocial profiles in adolescents, boys and girls, involved in different roles in dating violence and the usefulness of considering these specific profiles in the development of intervention programs.

Keywords

dating violence; victimization; adolescence; psychosocial profile. 
Actualmente, la violencia de pareja en adolescentes supone una importante problemática social debido a sus graves consecuencias y a su elevada prevalencia (Cortés-Ayala et al., 2015; Rey-Anacona, Mateus-Cubides \& Bayona-Arévalo, 2010; Viejo, 2014; Vizcarra \& Póo, 2011). En las parejas adolescentes se han observado niveles muy altos de violencia, sobre todo verbal-emocional, así como también de agresiones mutuas (FernándezFuertes \& Fuertes, 2010; Foshee et al., 2015). Su elevada prevalencia ha sido constatada por estudios realizados en diversos países como México (Cortés-Ayala et al., 2015), Colombia (Rey-Anacona, 2013), Chile (Vizcarra \& Póo, 2011) y España (Fernández-Fuertes \& Fuertes, 2010). En cuanto a las consecuencias que esta violencia tiene para los adolescentes, numerosas investigaciones muestran que estos presentan mayores dificultades a nivel personal y social, con mayor autoconcepto negativo, ansiedad, sintomatología depresiva, baja satisfacción con la vida, conductas inadecuadas de control de peso, abuso de sustancias, conductas sexuales inseguras e intentos de suicidio (Carrascosa, Cava \& Buelga, 2016; Exner-Cortens, 2014; Exner-Cortens, Eckenrode \& Rothman, 2013; Foshee et al., 2015; Shorey et al., 2012).

Una característica que diferencia la violencia en parejas adolescentes de la violencia entre parejas adultas, lo refleja el elevado número de agresiones mutuas existentes, y el doble rol de agresor y víctima que muchos adolescentes desempeñan en sus relaciones de pareja (Cava, Buelga \& Carrascosa, 2015; Viejo, Monks, Sanchez \& Ortega-Ruiz, 2016). No obstante, estas agresiones mutuas muestran importantes diferencias en función de la frecuencia con que se producen. Así, las agresiones mutuas ocasionales son las más habituales, y han sido vinculadas a formas torpes de cortejo así como la falta de experiencia de los adolescentes en este tipo de relaciones (Viejo, 2014; Viejo et al., 2016). Aunque evidentemente se trata de situaciones negativas, estas agresiones mutuas ocasionales estarían relacionadas con procesos transitorios de aprendizaje en un nuevo tipo de relaciones. Por el contrario, los adolescentes que desempeñan con frecuencia el doble rol agresor-víctima en sus relaciones de pareja, parecen presentar también mayores dificultades de adaptación en otros contextos sociales, como en las relaciones entre pares (Cava et al., 2015), y su perfil psicosocial podría ser más negativo. Aunque las agresiones mutuas en la pareja -frecuentes $u$ ocasionales- no son deseables, podrían existir importantes diferencias en el perfil psicosocial de los adolescentes que se integran en ambos grupos.

Por otra parte, una mejor comprensión de la violencia en parejas adolescentes parece requerir no sólo del análisis de la frecuencia con que las conductas violentas se producen, tal y como se ha sugerido en estudios previos (Rey-Anacona, 2013), sino también analizar qué características personales y sociales diferencian a los adolescentes, chicos y chicas, que desempeñan únicamente un rol de agresor o de víctima en relación con aquellos que desempeñan un doble rol de agresor-víctima. Un mayor conocimiento sobre el perfil psicológico y social de los chicos y chicas adolescentes con diferente rol en la violencia de pareja, puede ser de especial utilidad para el desarrollo de estrategias de intervención más efectivas.

Una de las características personales que convendría explorar en relación con el perfil psicosocial de los adolescentes con diferentes roles en la violencia de pareja es el autoconcepto. La adolescencia, es una etapa en la que se consolida la identidad (Exner-Cortens, 2014), se producen cambios importantes en la imagen que la persona tiene de sí misma a nivel físico, social y familiar, lo que implica además la necesidad de su análisis multidimensional (García, Musitu, Riquelme \& Riquelme, 2011). El autoconcepto ha sido relacionado tanto con variables indicativas de ajuste y bienestar psicosocial, como con conductas problemáticas, constatándose un autoconcepto más negativo en adolescentes que muestran mayores conductas agresivas (Gónzalez-Ortega, Echeburúa \& Corral, 2008). Asimismo, otras variables indicativas de bienestar general, como el ánimo depresivo y la satisfacción con la vida, relacionadas previamente con la violencia en 
adolescentes (Cava, Buelga \& Tomas, 2018; Estévez \& Jiménez, 2015; Exner-Cortens et al., 2013; Shorey et al., 2012), podrían contribuir a establecer un perfil psicosocial más claro de los diferentes roles que chicos y chicas adolescentes desempeñan en relación con la violencia de pareja.

Por otra parte, además de variables personales, también ciertas características del funcionamiento familiar pueden interferir en los adolescentes con distinto rol en la violencia de pareja. Ciertamente, estudios previos han mostrado la importancia que el contexto familiar tiene para el adecuado desarrollo psicosocial de los adolescentes y, en especial, la relevancia de la calidad de la comunicación entre padres e hijos en esta etapa evolutiva (Cava, Buelga \& Musitu, 2014; Estévez, Murgui, Moreno \& Musitu, 2007; Proctor, Linley \& Maltby, 2009). Mientras una comunicación familiar adecuada y la existencia de apoyo mutuo favorecen el bienestar psicosocial de los hijos, las conductas violentas, comunicación inadecuada y utilización del castigo están relacionadas con la violencia entre los adolescentes (Carrascosa, Cava \& Buelga, 2015; Estévez et al., 2007). Teniendo en cuenta la existencia de patrones de comunicación diferentes con la madre y con el padre (Parra \& Oliva, 2002), sería conveniente analizar por separado la comunicación que los hijos mantienen con ambos progenitores.

En este sentido, el objetivo de esta investigación, consistió en elaborar un perfil psicosocial de los chicos y chicas adolescentes españoles, con diferente rol en la violencia de pareja -agresores, víctimas, agresores-víctimas ocasionales, agresores-víctimas frecuentes y no implicados-, analizando sus posibles diferencias en autoconcepto -social, físico, académico, familiar y emocional-, satisfacción con la vida, ánimo depresivo y comunicación con la madre y con el padre. Como hipótesis inicial se plantea un perfil psicosocial más negativo en los adolescentes, tanto chicos como chicas, con un rol de víctima, agresor y agresorvíctima frecuente, en comparación con el perfil psicosocial de chicos y chicas no implicados y agresores-víctimas ocasionales.

\section{Método}

\section{Participantes}

La muestra inicial estuvo compuesta por 1078 adolescentes (545 chicas y 533 chicos), entre 12 y 19 años de edad $(M=14.45$, DT $=$ 1.62), estudiantes de Educación Secundaria y Bachillerato en cuatro centros educativos de la región de Valencia, España, tres de ellos públicos y uno privado-concertado. Todos los adolescentes participantes cumplimentaron los instrumentos sobre ajuste personal y familiar como parte de un estudio más amplio sobre la adolescencia. No obstante, solo los chicos y chicas adolescentes que tenían pareja en ese momento o que la habían tenido en los últimos 12 meses, cumplimentaron el instrumento que evalúa la violencia y victimización en la pareja. La muestra final estuvo formada por 672 adolescentes, 325 chicos (48.4\%) y 347 chicas (51.6\%). Su rango de edad se situó entre los 12 y 19 años $(M=14.45 ; D T=1.62)$, siendo similar la edad media de chicos $(M=14.36 ; D T=1.48)$ y chicas $(M=14.70 ; D T=1.65)$. Los porcentajes de adolescentes con 13 años (20.6\%), 14 años (20.9\%) y 15 años (22.0\%) son los más elevados, siendo menor el porcentaje de adolescentes con 12 años $(9.2 \%)$ y con 18 y 19 años (3.8\%).

\section{Instrumentos}

Escala de Violencia en Relaciones de Parejas Adolescentes CADRI (Wolfe et al., 2001; Fernández-Fuertes, Fuertes \& Pulido, 2006). La escala original consta de 70 ítems que evalúan tanto comportamientos violentos hacia la pareja, como experiencias de victimización. En este estudio se utilizaron tres subescalas de Violencia ejercida contra la pareja, con un total de 17 ítems: Violencia ejercida relacional: p.e., "Dije cosas a sus amigos sobre él/ella para ponerlos en su contra"; Violencia ejercida verbal-emocional: p.e., "Saqué a relucir algo malo que él/ella había hecho en el pasado" y Violencia ejercida física: p.e., "Le empujé o le zarandeé"; y tres subescalas de Violencia sufrida (17 ítems): Violencia sufrida 
relacional: p.e., "Trató de apartarme de mi grupo de amigos" Violencia sufrida verbal-emocional: p.e., "Me insultó con frases de desprecio" y Violencia sufrida física: p.e., "Me lanzó algún objeto". En esta escala se pide a los adolescentes que identifiquen con qué frecuencia se han producido en su relación de pareja, las situaciones descritas: nunca -esto no ha pasado en nuestra relación-, rara vez - 1 o 2 ocasiones-, a veces entre 3 y 5 veces- o con frecuencia -6 o más ocasiones-. En este estudio, el coeficiente de fiabilidad (alpha de Cronbach) para la escala total de violencia ejercida fue 0.91 y para las subescalas de violencia ejercida relacional, verbal-emocional y física fue, respectivamente, $0.65,0.85$ y 0.86 . La fiabilidad de la escala total de violencia sufrida fue 0.91 y para las subescalas de Violencia sufrida relacional, verbal-emocional y física fue $0.64,0.85$ y 0.82 , respectivamente.

Escala de Satisfacción con la Vida (Diener, Emmons, Larsen, \& Griffin, 1985; Atienza, Pons, Balaguer \& García-Merita, 2000). Esta escala evalúa con 5 ítems la satisfacción con la vida, aportando un índice general de satisfacción en términos de bienestar subjetivo: p.e., "No estoy contento/a con mi vida". Su coeficiente de fiabilidad (alpha de Cronbach) en el presente estudio fue 0.76 .

Escala de Sintomatología Depresiva (Herrero \& Meneses, 2006). Esta escala consta de 7 ítems: p.e., "Me costaba concentrarme en lo que estaba haciendo", que no evalúan depresión en sí misma sino sintomatología asociada pérdida de apetito, desamparo y desesperación, sentimientos de culpa e inutilidad, problemas de sueño, problemas de concentración-, aportando un indicador global de ánimo depresivo. Su coeficiente de fiabilidad (alpha de Cronbach) en este estudio fue 0.80 .

Escala de Autoconcepto-AF5- (García \& Musitu, 1999; García et al., 2011). Esta escala está compuesta por 30 ítems y mide cinco dimensiones del autoconcepto: Autoconcepto académico: p.e., "Soy un buen estudiante"; Autoconcepto social: p.e., "Tengo muchos amigos/as"; Autoconcepto emocional: p.e., "Me asusto con facilidad"; Autoconcepto familiar: p.e., "Me siento querido/a por mis padres"; y Autoconcepto físico: p.e., "Me considero elegante". Su consistencia interna (alpha de Cronbach) en este estudio fue 0.89 Autoconcepto académico; 0.71 Autoconcepto social; 0.68 Autoconcepto emocional; 0.80 Autoconcepto familiar y 0.77 Autoconcepto físico.

Escala de Comunicación Padres-Adolescentes (Barnes y Olson, 1985). Consta de dos escalas: una relativa a comunicación con la madre y otra referida a comunicación con el padre ambas desde la perspectiva del hijo/a-. Ambas escalas tienen 20 ítems, agrupados en dos dimensiones: Comunicación abierta y Problemas en la comunicación. La Comunicación abierta consta de 11 ítems: p.e., "Me presta atención cuando le hablo". La dimensión de Problemas en la comunicación consta de 9 ítems: p.e., "Intenta ofenderme cuando se enfada conmigo". La fiabilidad (alpha de Cronbach) en esta investigación para las subescalas de Comunicación abierta con el padre y la madre fue 0.91 para ambas; y para Problemas en la comunicación con el padre y la madre 0.70 , para ambas.

\section{Procedimiento}

La selección de la muestra, se realizó mediante muestreo por conglomerados, siendo las unidades de muestreo los centros educativos de Enseñanza Secundaria y Bachillerato de la región de Valencia, España. Una vez seleccionados los centros, se contactó telefónicamente y realizó una reunión con el equipo directivo asî como el profesorado para explicar el proyecto de investigación y solicitar su colaboración. Posteriormente, se envió a las familias una carta informativa sobre este proyecto y se les pidió su consentimiento. Una vez obtenidos los permisos, fueron varios investigadores previamente entrenados quienes aplicaron los instrumentos. Los adolescentes fueron informados de que su participación era voluntaria y anónima. El estudio cumplió con los valores éticos, respetando los principios fundamentales de la Declaración Helsinki (2008). 


\section{Análisis de datos}

En primer lugar, se calcularon las correlaciones entre todas las variables, en chicos y chicas por separado. Posteriormente, para analizar las diferencias en ajuste personal y familiar en función de los diferentes roles desempeñados por los adolescentes en la violencia de pareja, se establecieron cinco grupos de contraste: chicos y chicas no implicados en violencia ni victimización de pareja -adolescentes que respondieron 'nunca' a todos los ítems tanto de la escala total de violencia de pareja ejercida como de violencia de pareja sufrida-, agresores-víctimas frecuentes - con puntuaciones superiores a la media +1 DT tanto en la escala total de violencia de pareja ejercida como en la de violencia de pareja sufrida-, agresoresvíctimas ocasionales -puntuaciones inferiores a la media +1 DT en ambas escalas-, agresores puntuaciones superiores a la media +1 DT en violencia de pareja ejercida, pero inferiores a este punto de corte en violencia de pareja sufrida- y víctimas -puntuaciones superiores a la media +1 DT en violencia de pareja sufrida, pero inferiores a este punto de corte en violencia de pareja ejercida-. Se ha utilizado la media más 1 DT como punto de corte para clasificar a los chicos y chicas en estos grupos de contraste ya que dicho procedimiento ha sido considerado en estudios previos adecuados para identificar adolescentes con niveles elevados de conductas violentas (Marini, Dane, Bosacki, \& YLC-CURA, 2006). Una vez establecidos los grupos de contraste, se llevaron a cabo análisis multivariantes por separado con chicos y chicas. Todos los análisis se efectuaron utilizando el paquete estadístico SPSS-22.

\section{Resultados}

Los análisis de correlación de Pearson (ver Tabla 1) mostraron relaciones significativas entre la mayoría de variables, tanto en chicos como en chicas. Así, se observan en chicos $\mathrm{y}$ chicas correlaciones significativas positivas entre la violencia de pareja ejercida (VPE) y la violencia de pareja sufrida (VPS). En chicos y chicas, la VPE y la VPS correlacionan negativamente con la satisfacción con la vida, el autoconcepto familiar, y positivamente con el ánimo depresivo y los problemas de comunicación familiar $(p<0.05)$. Las diferentes formas (física, verbal-emocional y relacional) de VPE y VPS correlacionan en mayor medida con el autoconcepto familiar en las chicas, y con el autoconcepto emocional en los chicos $(p<0.05)$.

\section{Tabla 1}

Correlaciones entre todas las variables incluidas en el estudio (chicas en la parte superior de la diagonal)

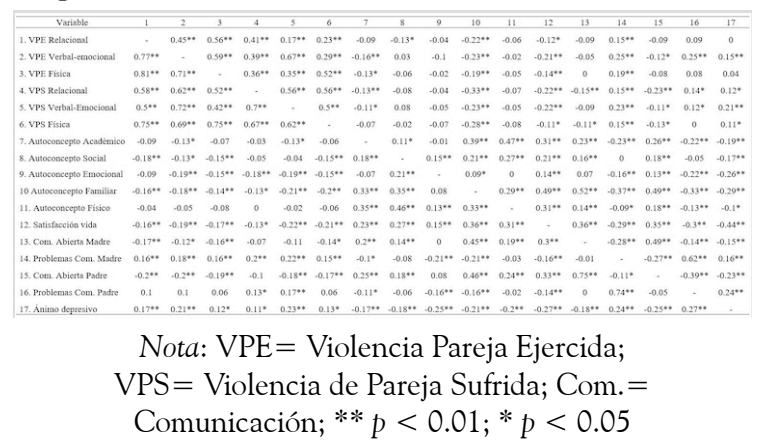

A continuación, en la Tabla 2 se muestran los datos relativos a la distribución de los adolescentes, chicos y chicas, en los cinco grupos de contraste establecidos en función de su diferente rol en la violencia de pareja. Los resultados de la prueba Chi-cuadrado de Pearson $=21.843(p<0.001)$ y de la prueba $\mathrm{V}$ de Cramer $=0.196 \quad(p<0.001)$ indicaron diferencias significativas entre chicos y chicas en su distribución dentro de estos grupos. En la tabla a continuación, se puede apreciar que la mayoría de adolescentes se sitúan en el grupo de 'agresor-víctima ocasional', siendo en este grupo superior el número de chicas. Por el contrario, el número de chicas que se perciben 'no implicadas' en ninguna forma de violencia -ni como agresoras ni como víctimas- es menor; así como también el de las que se integran en el grupo de 'agresorvíctima frecuente'. 
Tabla 2

Distribución de los adolescentes en función del sexo y de su diferente rol en la violencia de pareja

$\begin{array}{lrrrrr} & \begin{array}{c}\text { Número } \\ \text { implicados }\end{array} & \begin{array}{c}\text { Agresores-víctimas } \\ \text { ocasionales }\end{array} & \text { Víctimas } & \text { Agresores } & \begin{array}{c}\text { Agresores-víctimas } \\ \text { frecuentes }\end{array} \\ \text { Chicos } & 76(13.32) & 134(23.45) & 22(3.87) & 9(1.58) & 26(4.54) \\ \text { Chicas } & 50(8.75) & 205(35.90) & 15(2.62) & 15(2.63) & 19(3.34) \\ \text { Total } & 126(22.07) & 339(59.35) & 37(6.49) & 24(4.21) & 45(7.88)\end{array}$

Nota: Frecuencia (porcentaje).

Los resultados de los análisis multivariantes realizados con chicos (Tabla 3), indican diferencias significativas $(p<0.05)$ en satisfacción con la vida, ánimo depresivo, problemas de comunicación con la madre, comunicación abierta con el padre, autoconcepto académico, autoconcepto social y autoconcepto emocional entre los cinco grupos establecidos. No obstante, las pruebas a posteriori (prueba de Bonferroni) realizadas con la finalidad de conocer entre cuáles grupos en concreto existen estas diferentes, no hallaron diferencias significativas entre ninguna comparación entre grupos, dos a dos, en las variables de ánimo depresivo, problemas de comunicación con la madre y comunicación abierta con el padre. Además, en estas variables se observa que el tamaño del efecto $\left(\eta^{2}\right)$ es menor. Respecto a la satisfacción con la vida y el autoconcepto emocional, los resultados indican que los chicos con un rol frecuente de agresor-víctima tienen menor satisfacción con la vida que los no implicados y los que desempeñan este rol de agresor-víctima de forma ocasional $\left(p=0.003 ; \eta^{2}=0.059\right)$, y su autoconcepto emocional es significativamente más bajo que el de los adolescentes no implicados $\left(p=.004 ; \eta^{2}=.056\right)$. Los chicos agresores muestran menor autoconcepto social que los agresores-víctimas ocasionales $(\mathrm{p}<0.000$; $\eta^{2}=0.068$ ), y los chicos víctimas tienen menor autoconcepto académico que los no implicados $\left(p=0.020 ; \eta^{2}=0.047\right)$.

En relación a las diferencias entre grupos en chicas, en la Tabla 3 se observa que las chicas no implicadas y con un rol ocasional de agresora-víctima tienen mayor satisfacción con la vida, menor ánimo depresivo, más comunicación abierta con el padre y mayor autoestima familiar que las chicas víctimas de esta violencia $\left(p<0.000 ; \quad \eta^{2}=0.083 ; \quad p<0.000 ; \quad \eta^{2}=0.071 ;\right.$ $\left.p=0.016 ; \quad \eta^{2}=0.045 ; \quad p<0.000 ; \quad \eta^{2}=0.099\right)$. Además, muestran también mayor satisfacción con la vida y mayor autoestima familiar que las chicas con rol frecuente de agresora-víctima. Las chicas no implicadas presentan menores problemas de comunicación con el padre que las agresoras $\left(p=0.002 ; \eta^{2}=0.061\right)$, comunicación más abierta con la madre que las víctimas $\left(p<0.029 ; \eta^{2}=0.046\right)$ y menos problemas de comunicación con la madre que el resto de chicas adolescentes $\left(p<0.000 ; \eta^{2}=0.089\right)$, mostrando una comunicación familiar más positiva. Estas chicas no implicadas de ninguna forma en la violencia de pareja, ni como agresoras ni como víctimas, muestran también mayor autoconcepto académico que las chicas víctimas y las que desempeñan un rol de agresora-víctima, tanto de forma frecuente como ocasional $(p=0.001$; $\left.\eta^{2}=0.053\right)$.

\section{Tabla 3}

Medias y desviaciones típicas en satisfacción con la vida, ánimo depresivo, comunicación familiar y autoconcepto en chicos y chicas en función de su implicación en la violencia de pareja

\begin{tabular}{|c|c|c|c|c|c|c|c|}
\hline & \multicolumn{7}{|c|}{ VIOLENCIA DE PAREJA } \\
\hline & No implicados & $\begin{array}{c}\text { Agresiones } \\
\text { muttuas } \\
\text { ocasionales }\end{array}$ & $\begin{array}{l}\text { Victimas } \\
\text { pasivas }\end{array}$ & $\begin{array}{l}\text { Agresores } \\
\text { no victimas }\end{array}$ & $\begin{array}{c}\text { Agresiones } \\
\text { mutuas } \\
\text { frecuentes }\end{array}$ & & \\
\hline & \multicolumn{5}{|c|}{ Hombres } & $p$ & $\eta^{2}$ \\
\hline Satisfacción vida & $18.21(4.3) \mathrm{a}$ & $18.35(3.65) \mathrm{a}$ & $16.09(3.36)$ & $17.33(5.09)$ & & 0 & 0.05 \\
\hline Animo depresivo & $12.35(4.08)$ & $13.52(3.92)$ & $14.95(3.94)$ & $14.33(3.46)$ & $14.33(3.22)$ & 0.03 & 0.04 \\
\hline \multicolumn{8}{|l|}{ Comunicación madre } \\
\hline Abierta & $43.03(10.33)$ & $41.6(9.85)$ & $38.43(10.33)$ & $40.44(8.06)$ & $39.64(12.6)$ & 0.35 & 0.02 \\
\hline - Problemas & $19.75(6.5)$ & $21.85(5.57)$ & $23.38(5.75)$ & $23.78(5.11)$ & $23.52(6.17)$ & 0.01 & 0.04 \\
\hline \multicolumn{8}{|l|}{ Comunicación pac } \\
\hline $\begin{array}{l}\text { Abierta } \\
\text { Problemas }\end{array}$ & 41.79( & $38.89(10.18)$ & $34.65(10.24)$ & 34: & 91) & 0.02 & 0.04 \\
\hline \multirow{2}{*}{\multicolumn{6}{|c|}{ 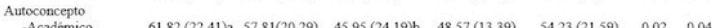 }} & & 0.03 \\
\hline & $61.82(22.41)_{\mathrm{a}}$ & $57.81(20.29)$ & $45.95(24.19) \mathrm{b}$ & 48.57 (13.39) & $54.23(21.59)$ & 0.02 & 0.04 \\
\hline -Social & (1) & $80.37(11.64) \mathrm{a}$ & $72.62(16.34)$ & $63(17$ & $72.46(17.29)$ & 0 & 0.06 \\
\hline -Emocional & $63.67(18.45) \mathrm{a}$ & $56.84(17.22)$ & $61.42(13.24)$ & $62.93(16.52)$ & $49.28(20.25) \mathrm{b}$ & 0 & 0.05 \\
\hline Familiax & $84.52(16.18)$ & $83.36(15.64)$ & $75.66(17.67)$ & $80.44(20.22)$ & $76.06(18.91)$ & 0.05 & 0.03 \\
\hline -Fisico & $68.34(18.58)$ & $73.49(15.59)$ & $65.02(20.21)$ & $72.63(9.62)$ & $67.54(18.62)$ & 0.08 & 0.03 \\
\hline & \multicolumn{5}{|c|}{ Mujeres } & $p$ & $\eta^{2}$ \\
\hline Satisfacción vida & $18.53(3.82) \mathrm{a}$ & $17.61(3.95) \mathrm{a}$ & $13.53(3.85) \mathrm{b}$ & $16.2(4.21)$ & $15.05(4.06) \mathrm{b}$ & 0 & 0.08 \\
\hline Animo depresivo & $14.14(4.95) \mathrm{b}$ & $15.11(4.52) \mathrm{b}$ & $19.71(4.93) \mathrm{a}$ & $17.07(4.89)$ & $17.42(4.05)$ & 0 & 0.07 \\
\hline \multicolumn{8}{|l|}{ Comunicación madre } \\
\hline -Abierta & $45.2(8.52) \mathrm{a}$ & $42.63(8.92)$ & $36.93(9.31) \mathrm{b}$ & $40.6(7.69)$ & $44.16(10.85)$ & 0.02 & 0.04 \\
\hline - Problema & $19.49(5.81) \mathrm{b}$ & $22.41(5.67) \mathrm{a}$ & $25.86(3.84) \mathrm{a}$ & $25.4(3.64) \mathrm{a}$ & $25.21(6.82) \mathrm{a}$ & 0 & 0.08 \\
\hline Comunicación padre & & & & & & 0.01 & 0.04 \\
\hline $\begin{array}{l}\text {-Abierta } \\
\text {-Problemas }\end{array}$ & $\begin{array}{l}39.65(9.72) \mathrm{a} \\
20.24(5.9) \mathrm{b}\end{array}$ & $\begin{array}{l}37.73(9.67) \mathrm{a} \\
22.77(5.64)\end{array}$ & $\begin{array}{l}29.58(9.09) b \\
23.92(4.2)\end{array}$ & $\begin{array}{l}35.27(8.41) \\
26.33(4.76) \mathrm{a}\end{array}$ & $\begin{array}{l}35.06(10.59) \\
24.24(5.66)\end{array}$ & 0 & 0.06 \\
\hline \multicolumn{8}{|c|}{ 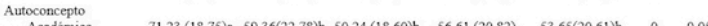 } \\
\hline -Académico & $71.23(18,75) \mathrm{a}$ & $59.36(22.78) \mathrm{b}$ & $50.24(18.60) b$ & 56.61 & 53.65( & 0 & 0.05 \\
\hline -Social & $69.13(18.13)$ & $75.37(15.08)$ & $69.11(17.3)$ & $70.82(16.78)$ & $75.41(14.37)$ & 0.08 & 0.02 \\
\hline -Emocional & $47.46(21.07)$ & $48.26(19.13)$ & $35.18(22.35)$ & $40.66(17.01)$ & $46.73(14.13)$ & 0.1 & 0.02 \\
\hline -Familiar & $88.01(13.64) \mathrm{a}$ & $82.29(15.91) \mathrm{a}$ & $64.84(23.41) \mathrm{b}$ & $75.36(20.51)$ & $70.64(25.61) \mathrm{b}$ & 0 & 0.09 \\
\hline Fisico & $57.24(20.32)$ & $56.82(20.25)$ & $50.88(18.5)$ & $57.36(14.86)$ & $51.40(20.24)$ & 0.63 & 0.01 \\
\hline
\end{tabular}

| Universitas Psychologica | V. I7 | No.3 | 2018 | 


\section{Discusión}

El objetivo de este estudio consistió en elaborar un perfil psicosocial de los adolescentes españoles, chicos y chicas, con diferente rol en la violencia de pareja -agresor, víctima, agresorvíctima ocasional, agresor-víctima frecuente y no implicado-, analizando sus posibles diferencias en satisfacción con la vida, ánimo depresivo, autoconcepto y comunicación familiar. En primer lugar, y respecto a la prevalencia con que estos roles son desempeñados por los adolescentes españoles, los resultados obtenidos señalan que la mayor parte mantienen un rol de agresorvíctima ocasional, lo que confirma tanto la elevada prevalencia de las agresiones mutuas en parejas adolescentes (Fernández-Fuertes \& Fuertes, 2010; Fernández-Fuertes et al., 2011), como el hecho de que las agresiones mutuas ocasionales sean las más habituales (Cava et al., 2015). Por otra parte, este estudio aporta además resultados novedosos sobre el diferente perfil psicosocial de los adolescentes con rol de agresor-víctima ocasional y con rol de agresorvíctima frecuente, siendo más negativo el perfil psicosocial de este último grupo. Los chicos y chicas con un rol frecuente de agresor-víctima, muestran una menor satisfacción con la vida, variable considerada como un potente indicador de ajuste psicosocial y como posible variable mediadora en el afrontamiento adecuado de situaciones estresantes (Proctor et al., 2009), por lo que la diferencia entre ambos grupos en esta variable es de especial relevancia.

Además, los resultados obtenidos destacan, en general un perfil psicosocial más positivo en los chicos/chicas no implicados y con un rol de agresor-víctima ocasional, en comparación con aquellos con un rol de agresor, de víctima o de agresor-víctima frecuente, confirmando así la hipótesis inicial. Este perfil más positivo de los adolescentes con un rol ocasional de agresorvíctima, supone un apoyo para planteamientos previos que han sugerido una vinculación de las agresiones mutuas ocasionales con la falta de experiencia previa de los adolescentes en relaciones de pareja (Cava et al., 2015; Viejo, et al., 2016). Por otra parte, se observaron también algunas diferencias interesantes entre el perfil psicosocial de los chicos y las chicas con diferentes roles. Así, en los chicos, son los que desempeñan un rol de agresor-víctima frecuente los que tienen menor satisfacción con la vida y menor autoconcepto emocional. Sin embargo, en las chicas, son las víctimas de violencia de pareja las que tienen el perfil psicosocial más negativo, mostrando no sólo menor satisfacción con la vida, sino también mayor ánimo depresivo, menor autoconcepto académico y familiar y más dificultades en la comunicación con ambos progenitores. Tanto las chicas que son víctimas como las que tienen un rol frecuente de agresoravíctima manifiestan un importante malestar psicológico y, a diferencia de los chicos, mayores dificultades en la comunicación familiar. Estos resultados confirmarían que, aunque chicos y chicas se encuentren implicados en la violencia de pareja, las consecuencias parecen ser más graves e impactantes para las chicas (Straus, 2011).

Por otra parte, respecto a la comunicación familiar, en estudios previos se ha constatado que las chicas tienen un mayor vínculo con el contexto familiar y que la familia tiene una elevada influencia en su satisfacción con la vida (Cava et al., 2014), lo que podría estar relacionado con los mayores problemas de comunicación con la madre y el menor autoconcepto familiar observado en las chicas con un rol de agresora-víctima frecuente. Así, si bien un entorno familiar positivo y una comunicación familiar abierta favorecen el ajuste psicosocial de chicos y chicas adolescentes (Cava et al., 2014; Foshee et al., 2015), su importancia en relación con la violencia de pareja podría ser mayor para las chicas, y debería ser un objetivo prioritario de las intervenciones dirigidas a la prevención de la violencia en chicas adolescentes. Estas diferencias entre chicos y chicas podrían explicarse por los diferentes estilos de socialización que ambos progenitores utilizan dependiendo del sexo de sus hijos (Garaigordobil \& Aliri, 2012). Asimismo, sería también interesante explorar su posible relación con los distintos estilos de socialización utilizados con chicos y chicas postulados por la Teoría 
de las dos culturas (Maccoby, 1998), en la que se destaca una mayor socialización de los chicos en la cultura de la violencia y en una mayor utilización de estrategias violentas de resolución de conflictos. Estas cuestiones deberán ser exploradas con mayor profundidad en futuras investigaciones.

En cuanto a las limitaciones de este trabajo, es necesario destacar su naturaleza transversal, que no permite establecer relaciones causales entre las variables analizadas. Así, las diferencias observadas entre los adolescentes con diferentes roles en la violencia de pareja, podrían ser, tanto consecuencia de la violencia, como factores de riesgo previos que acrecentarían su vulnerabilidad a implicarse en situaciones de violencia de pareja, ya sea como agresores, víctimas o desempeñando ambos roles. Son, por tanto, necesarios estudios longitudinales que permitan un mayor conocimiento sobre estas relaciones. No obstante, y a pesar de estas limitaciones, este trabajo aporta resultados nuevos e interesantes sobre el perfil psicosocial de los chicos y chicas con diferentes roles en la violencia de pareja. Los resultados obtenidos destacan el perfil psicosocial más negativo de los chicos y chicas con un rol frecuente de agresorvíctima y de las chicas víctimas de violencia de pareja. Debido a las mayores dificultades en la comunicación familiar, constatadas en las chicas implicadas en la violencia de pareja, esta variable debería incluirse en los programas de prevención con adolescentes. Las intervenciones en este ámbito deberían dirigirse prioritariamente a los chicos y chicas con un rol frecuente de agresorvíctima y a las chicas víctimas, tanto para evitar las consecuencias negativas que esta violencia implica, como para prevenir que las dinámicas interpersonales violentas se perpetúen en sus futuras relaciones de pareja en edad adulta.

\section{Agradecimientos}

Esta investigación se ha elaborado en el marco del Proyecto de Investigación ACIF/2015/198, "Intervención en violencia escolar y violencia de pareja en adolescentes", subvencionado por la Conselleria de Educació, Cultura i Esport de la Generalitat Valenciana dentro del Programa $\mathrm{VALi}+\mathrm{d}$ para investigadores en formación.

\section{Referencias}

Atienza, F. L., Pons, D., Balaguer, I. \& García-Merita, M. (2000). Propiedades psicométricas de la escala de satisfacción con la vida en adolescentes. Psicothema, 12(2), 314-319. Recuperado de http://www .psicothema.com/psicothema.asp?id=296

Barnes, H. L. \& Olson, D. H. (1985). Parent-adolescent communication and the circumplex model. Child Development, 56(2), 438-447. https://doi.org/10.2307/11 29732

Carrascosa, L., Cava, M.J. \& Buelga, S. (2015). Actitudes hacia la autoridad y violencia entre adolescentes: Diferencias en función del sexo. Suma Psicológica, 22(2), 102-109. https://doi.org/10.1016/j.sumpsi.2 015.08 .003

Carrascosa, L, Cava, M.J., \& Buelga, S. (2016). Ajuste psicosocial en adolescentes víctimas frecuentes y víctimas ocasionales de violencia de pareja. Terapia Psicológica, 34(2), 93-102. http://doi.org/10.4067/S071 8-48082016000200002

Cava, M.J., Buelga, S., \& Carrascosa, L. (2015). Violencia física y psicológica ejercida en parejas adolescentes: Relación con el autoconcepto y la violencia entre iguales. Psicología Conductual, 23(3), 429-446. Recuperado de https://search.proquest.com/openview/e 40a3a4f288a6552b32115dc64806e8e/1?pq - origsite $=$ gscholar\&cbl $=966333$

Cava, M.J., Buelga, S., \& Musitu, G. (2014). Parental communication and life satisfaction in adolescence. The Spanish Journal of Psychology, 17(E98), 1-8. https:// doi.org/10.1017/sjp.2014.107

Cava, M. J., Buelga, S., \& Tomás, I. (2018). Peer Victimization and Dating Violence Victimization: The Mediating Role of Loneliness, Depressed Mood, and 
Life Satisfaction. Journal of Interpersonal Violence. Advance online publication. http: //doi.org/10.1177/0886260518760013.

Cortés-Ayala, L., Flores, M., Bringas, C., Rodríguez-Franco, L., López-Cepero, J. \& Rodríguez, J. (2015). Relación de maltrato en el noviazgo de jóvenes mexicanos. Análisis diferencias por sexo y nivel de estudios. Terapia Psicológica, 33(1), 5-12. Recuperado de http://www.redalyc.org/arti culo.oa? id $=78539320001$

Declaración de Helsinki (2008) Principios éticos para las investigaciones médicas en seres humanos. Declaración aprobada en la 59a Asamblea General de la Asociación, celebrada en Seúl, Corea. http://bvs.sld.cu/ revistas/recursos/helsinki.pdf

Diener, E., Emmons, R., Larsen, R. J., \& Griffin, S. (1985). The Satisfaction with Life Scale. Journal of Personalitiy Assessment, 49(1), 71-75. Recuperado de https://www.ncbi.nl m.nih.gov/pubmed/16367493

Estévez, E. \& Jiménez, T. I. (2015). Conducta agresiva y ajuste personal y escolar en una muestra de estudiantes adolescentes españoles. Universitas Psychologica, 14(1), 111-124. https://doi.org/10.11144/Javerian a.upsy14-1.caap

Estévez, E., Murgui, S., Moreno, D., \& Musitu, G. (2007). Estilos de comunicación familiar, actitud hacia la autoridad institucional y conducta violenta del adolescente en la escuela. Psicothema, 19(1), 108-113. Recuperado de http://www.psicothema.co $\mathrm{m} / \mathrm{pdf} / 3335 . \mathrm{pdf}$

Exner-Cortens, D. (2014). Theory and teen dating violence victimization: considering adolescent development. Developmental Review, 34(2), 168-188. https://doi.org/10.1 016/j.dr.2014.03.001

Exner-Cortens, D., Eckenrode, J., \& Rothman, E. (2013). Longitudinal associations between teen dating violence victimization and adverse health outcomes. Pediatrics, 131(1), 71-78. https://doi.org/10.1542/peds.2012-1 029

Fernández-Fuertes, A. A. \& Fuertes, A. (2010). Physical and psychological aggression in dating relationships of Spanish adolescents: Motives and consequences. Child Abuse E3 Neglect, 34(3), 183-191. https:// doi.org/10.1016/j.chiabu.2010.01.002

Fernández-Fuertes, A. A., Fuertes, A., \& Pulido, R. F. (2006). Evaluación de la violencia en las relaciones de pareja de los adolescentes. Validación del Conflict in Adolescent Dating Relationships Inventory (CADRI)-versión española. International Journal of Clinical and Health Psychology, 6(2), 339-358. Recuperado de http://www. aepc.es/ijchp/articulos_pdf/ijchp-181.pdf

Fernández-Fuertes, A. A., Orgaz, B., \& Fuertes, A. (2011). Características del comportamiento agresivo en las parejas de los adolescentes españoles. Psicología Conductual, 19(3), 501-522. Recuperado de https://search.proquest.com/docview/92 7580538/fulltextPDF/6626AC3872524C5 $\mathrm{CPQ} / 1$ ? accountid $=14777$

Foshee, V. A., Reyes, L. M., Tharp, A. T., Chang, L.Y., Ennett, S.T., ... Suchindran, C. (2015). Shared longitudinal predictors of physical peer and dating violence. Journal of Adolescent Health, 56(1), 106-112. https://d oi.org/10.1016/j.jadohealth.2014.08.003

Garaigordobil, M. \& Aliri, J. (2012). Parental socialization styles, parents' educational level and sexist attitudes in adolescence. The Spanish Journal of Psychology, 15(2), 592-603. Recuperado de https://www.ncbi. nlm.nih.gov/pubmed/22774433

García, F. \& Musitu, G. (1999). Manual AF-5: Autoconcepto Forma-5. Madrid: TEA.

García, F., Musitu, G., Riquelme, E., \& Riquelme, P. (2011). A confirmatory factor analysis of the "Autoconcepto Forma 5" questionnaire in young adults from Spain and Chile. The Spanish Journal of Psychology, 14(2), 648-658. https://doi.org/10.5209/rev_SJOP .2011.v14.n2.13

González-Ortega, I., Echeburúa, E., \& Corral, P. (2008). Variables significativas en las relaciones violentas en parejas jóvenes: una revisión. Psicología Conductual, 16(2), 207-225. Recuperado de http://www.ehu.e us/echeburua/pdfs/04GONZALEZ.pdf 
Herrero, J. \& Meneses, J. (2006). Short Webbased versions of the perceived stress (PSS) and Center for Epidemiological StudiesDepression (CESD) Scales: a comparison to pencil and paper responses among Internet users. Computers in Human Behavior, 22 (5), 830-846. https://doi.org/10.1016/j.chb.200 4.03.007

Maccoby, E. E. (1998). The two sexes: Growing apart, coming together. Cambridge, MA: Harvard University Press.

Marini, Z.A., Dane, A.V., Bosack, S.L. \& YLC-CURA (2006). Direct and indirect bully-victims: Differential psychosocial risk factors associated with adolescents involved in bullying and victimization. Aggressive Behavior, 32 (6), 551-569. https:// doi.org/10.1002/ab.20155

Parra, A. \& Oliva, A. (2002). Comunicación y conflicto familiar durante la adolescencia. Anales de Psicología, 18(2), 215-231. Recuperado de http://www.redalyc.org/pdf/ 167/16718202.pdf

Proctor, C. L., Linley, P. A. \& Maltby, J. (2009). Youth life satisfaction: A review of the literature. Journal of Happiness Studies, 10(5), 583-630. https://doi.org/10.1007/s10 902-008-9110-9

Rey-Anacona, C. A. (2013). Prevalencia y tipos de maltrato en el noviazgo en adolescentes y adultos jóvenes. Terapia Psicológica, 31 (4), 143-154. Recuperado de http://www.redaly c.org/pdf/785/78526609001.pdf

Rey-Anacona, C. A., Mateus-Cubides, A. M., \& Bayona-Arévalo, P.A. (2010). Malos tratos ejercidos por adolescentes durante el noviazgo: diferencias por sexo. Revista Mexicana de Psicología, 27(2), 169-181. Recuperado de http://www.redalyc.org/pdf/ 2430/243016324006.pdf

Shorey, R. C., Temple, J. R., Febres, J., Brasfield, H., Sherman, A. E., \& Stuart, G. L. (2012). The consequences of perpetrating psychological aggression in dating relationships a descriptive investigation. Journal of Interpersonal Violence, 27(15), 2980-2998. https://doi.org /10.1177/0886260512441079
Straus, M.A. (2011). Gender symmetry and mutuality in perpetration of clinical-level partner violence: Empirical evidence and implications for prevention and treatment. Aggression and Violent Behavior, 16(4), 279-288. https://doi.org/10.1016/j.avb.201 1.04 .010

Viejo, C. (2014). Violencia física en las relaciones sentimentales adolescentes: hacia la comprensión del fenómeno. Infancia y Aprendizaje, 37(4), 785-815. https://doi.org $/ 10.1080 / 02103702.2014 .977110$

Viejo, C., Monks, C. P., Sanchez, V., \& OrtegaRuiz, R. (2016). Physical dating violence in Spain and the United Kingdom and the importance of relationship quality. Journal of Interpersonal Violence, 31(8), 1453-1475. https://doi.org/10.1177/088626 0514567963

Vizcarra, M. B. \& Póo, A. M. (2011). Violencia de pareja en estudiantes universitarios del sur de Chile. Universitas Psychologica, 10(1), 89-98. Recuperado de http://www.scielo.or g.co/pdf/rups/v10n1/v10n1a08.pdf

Wolfe, D.A., Scott, K., Reitzel-Jaffe, D., Wekerle, C., Grasley, C., \& Pittman, A.L. (2001). Development and validation of the conflict in adolescent dating relationships inventory. Psychological Assessment, 13(2), 277-293. Recuperado de https://www.ncbi. nlm.nih.gov/pubmed/11433803

\section{Notas}

* Artículo de investigación. 